Article

\title{
Development of a Honey Bee RNA Virus Vector Based on the Genome of a Deformed Wing Virus
}

\author{
Eugene V. Ryabov ${ }^{1, * \mathbb{C}}$, Krisztina Christmon ${ }^{1,2}$, Matthew C. Heerman ${ }^{1}$, \\ Francisco Posada-Florez ${ }^{1}$, Robert L. Harrison ${ }^{3}$, Yanping Chen ${ }^{1}$ and Jay D. Evans ${ }^{1}$ \\ 1 USDA, Agricultural Research Service, Bee Research Lab, Beltsville, MD 20705, USA; \\ kriszch@umd.edu (K.C.); matthew.heerman@usda.gov (M.C.H.); francisco.posada@usda.gov (F.P.-F.); \\ judy.chen@usda.gov (Y.C.); jay.evans@usda.gov (J.D.E.) \\ 2 Department of Entomology, University of Maryland, College Park, MD 20742, USA \\ 3 USDA, Agricultural Research Service, Invasive Insect Biocontrol and Behavior Laboratory, \\ Beltsville, MD 20705, USA; robert.1.harrison@usda.gov \\ * Correspondence: eugene.ryabov@gmail.com
}

Received: 20 February 2020; Accepted: 26 March 2020; Published: 28 March 2020

check for updates

\begin{abstract}
We developed a honey bee RNA-virus vector based on the genome of a picorna-like Deformed wing virus (DWV), the main viral pathogen of the honey bee (Apis mellifera). To test the potential of DWV to be utilized as a vector, the $717 \mathrm{nt}$ sequence coding for the enhanced green fluorescent protein (eGFP), flanked by the peptides targeted by viral protease, was inserted into an infectious cDNA clone of DWV in-frame between the leader protein and the virus structural protein VP2 genes. The in vitro RNA transcripts from egfp-tagged DWV cDNA clones were infectious when injected into honey bee pupae. Stable DWV particles containing genomic RNA of the recovered DWV with egfp inserts were produced, as evidenced by cesium chloride density gradient centrifugation. These particles were infectious to honey bee pupae when injected intra-abdominally. Fluorescent microscopy showed GFP expression in the infected cells and Western blot analysis demonstrated accumulation of free eGFP rather than its fusions with DWV leader protein (LP) and/or viral protein (VP) 2. Analysis of the progeny egfp-tagged DWV showed gradual accumulation of genome deletions for egfp, providing estimates for the rate of loss of a non-essential gene an insect RNA virus genome during natural infection.
\end{abstract}

Keywords: honey bee; deformed wing virus; RNA virus vector; invertebrate virus; virus evolution; pollination; food security

\section{Introduction}

Vectors based on the genomes of RNA viruses of animals [1,2] or plants [3,4] have been widely used in research and biotechnology since the 1990s. RNA vectors provide transient peptide expression at high levels for research and industrial protein production [4] without the generation of transgenic hosts. Moreover, RNA virus vectors allow the expression of proteins, which could be toxic or affect host development, a complication for transgenic approaches. Another unique feature of RNA virus vectors is their use for the induction of RNA interference (RNAi) due to the generation of double-stranded (ds) RNA replication intermediates [5]. Importantly, virus-based vectors that express green fluorescent protein (GFP) or other reporters enable profound studies of viruses and their interactions with hosts [6].

Insect-specific RNA virus vectors have been designed using genomes of the arboviruses, members of Flavivirus, Alphavirus and Nodavirus groups [1,7,8]. Currently, there are no RNA vectors based on genomes of viruses naturally infecting honey bees. While GFP-tagged vector based on a cloned Sindbis virus, a member of the Alphavirus group, was successfully used in honey bees [9], the development of 
reporters tied to an infective honey bee virus would provide a novel system to investigate virus and host interactions.

In this study, we develop a virus expression system for the widespread virus DWV type A [10] using a recently designed infectious cDNA clone of genomic RNA of this virus [11]. We identified a site in the DWV genomic RNA insertion into which an enhanced green fluorescent protein (eGFP)-coding sequence [12], corresponding to 7\% of the DWV RNA genome, did not affect virus replication. We demonstrated the encapsidation of DWV RNA following egfp gene insertion and showed the expression of the egfp reporter in the cells of honey bee pupae infected with the egfp-tagged DWV RNA vectors by using fluorescent microscopy and Western blot. Analysis of the genetic stability of the DWV vector provided novel insights into the ability of RNA viruses to retain non-essential sequences, contributing to a better understanding of their evolution.

\section{Materials and Methods}

\subsection{Construction of the egfp-Tagged DWV cDNA Clones and Generation of the Clone-Derived Viruses}

The full-length infectious cDNA clone of DWV type A, pDWV-304 (GenBank accession number MG831200) [11] was used to design eGFP-expressing constructs, pDWV-L-GFP and pDWV-S-GFP (Figure 1; Text S1). Cloning included the generation of sections of DWV cDNA with egfp insertions at the leader protein-viral protein 2 (LP-VP2) border by overlap extension polymerase chain reaction (PCR) and subcloning, using pDWV-304 [11] and pAcP(+)IE1-eGFP [13] as the templates for DWV and egfp amplification, respectively; the primers are listed in Table S1. The cloning procedure is described in detail in Text $\mathrm{S} 1$.

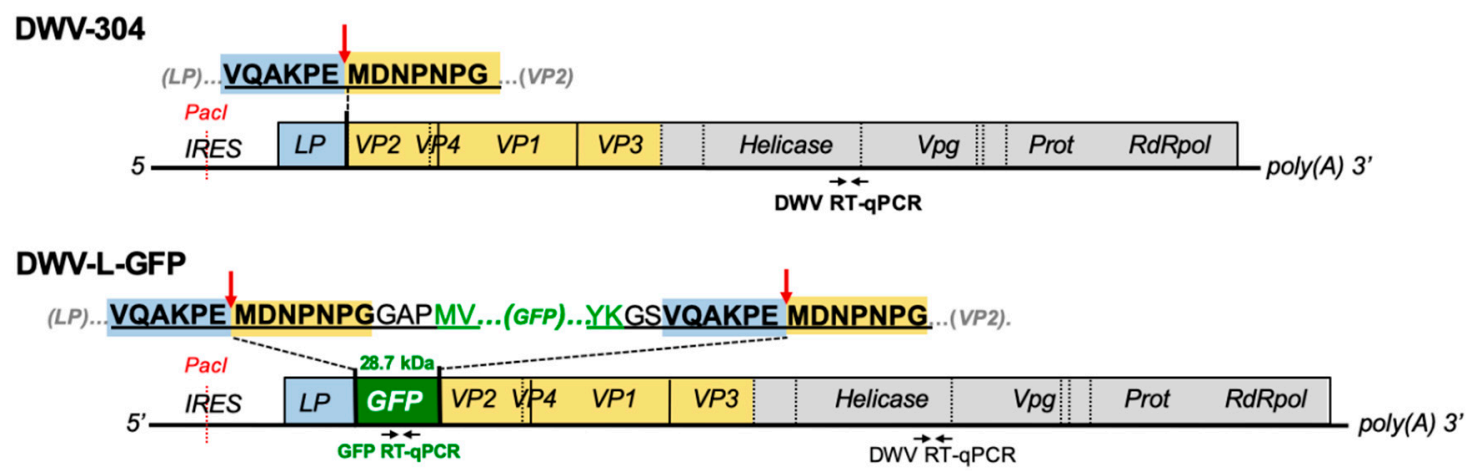

\section{DWV-S-GFP}

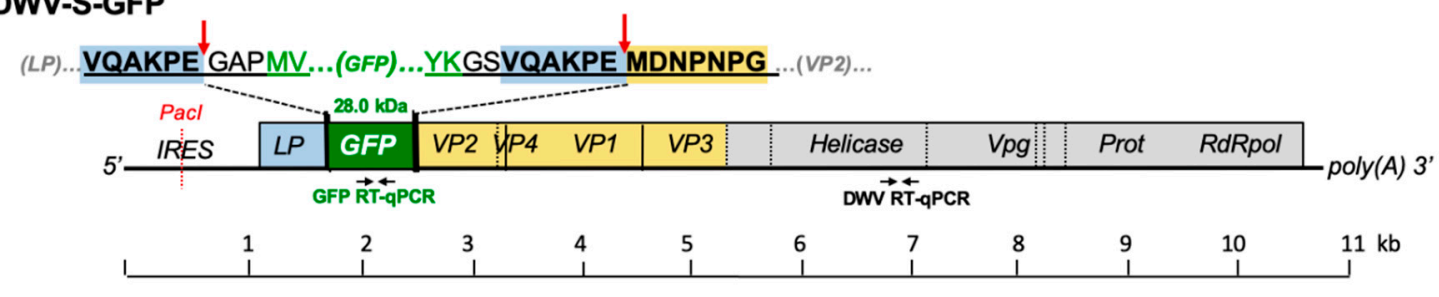

Figure 1. Design of the green fluorescent protein (GFP)-tagged cDNA clones of Deformed wing virus (DWV). Sequences of the peptide linking LP-VP2 and the GFP-flanking peptides are shown above the genetic maps. Arrows indicate the tentative cleavage peptides targeted by the DWV $3 \mathrm{C}$ protease, underscored are DWV-derived peptides. Positions of the DWV- and GFP-specific RT-qPCR primers are indicated. DWV, deformed wing virus; LP, leader protein; GFP, green fluorescent protein; Prot, viral 3C protease; RdRpol, RNA-dependent RNA polymerase; RT-PCR, reverse-transcription PCR; VP, structural viral protein; $\mathrm{Vpg}$, genome-linked protein.

The egfp-tagged DWV cDNA plasmid constructs were linearized using the PmeI restriction site located at the $3^{\prime}$ end of DWV cDNA, downstream of the poly A sequence, to produce the templates for a full-length 10.9-kb transcript. The in vitro RNA transcripts from pDWV-L-GFP, pDWV-S-GFP and the 
parental pDWV-304 were produced using the HiScribe T7 High Yield RNA Synthesis Kit (New England Biolabs) according to the manufacturer's instructions. After $3 \mathrm{~h}$ of incubation at $+37^{\circ} \mathrm{C}$, the DNA templates were digested using TURBO DNase (Life Technologies). The in vitro RNA transcripts were purified using the RNeasy mini kit (Qiagen), $13.2 \times 10^{12}$ copies in $10 \mu \mathrm{L}$ of phosphate buffered saline (PBS), or $10 \mu \mathrm{L}$ of PBS were injected intra-abdominally into the hemolymph of honey bee pupae at the pink eye stage that were not exposed to Varroa mite feeding, using syringes with a 0.3-mm needle G31 (BD Micro-Fine) as described previously [11]. The extracts containing DWV-L-GFP and DWV-S-GFP virus particles were produced by homogenizing individual transcript-injected pupae with $1.5 \mathrm{~mL}$ of PBS at $72 \mathrm{hpi}$ and subjected to three cycles of freeze-thawing, preparation included clarification by centrifugation at $3000 \times g$ for $5 \mathrm{~min}$ and filtration through a $0.22 \mu \mathrm{m}$ nylon filter (Millipore). The DWV concentration in the extracts was quantified by RT-qPCR, and the extracts were stored at $-80^{\circ} \mathrm{C}$ before they were used.

\subsection{Honey Bees}

Honey bee pupae were obtained from strong colonies with low (less than 1\%) Varroa mite infestation rate from the apiaries located in the USDA Bee Research Laboratory (BRL) in Beltsville, Maryland (sourced in September 2019), and in the USDA Honey Bee Breeding, Genetics and Physiology Research Laboratory in Baton Rouge, Louisiana USA (sourced in November 2019). Pupae at the pink-eyed stage were pulled out of cells using soft tweezers $3 \mathrm{~h}$ before their use in the injection experiments, which were carried out as described previously [11]. Newly emerged honey bees with deformed wings sourced in Maryland in 2019 were used as the source of the wild-type DWV for Figure 7B.

\subsection{RT-qPCR and Virus Progeny Analysis}

Total RNA extracted from individual honey bee pupae using Trizol reagent (Ambion) or $\mathrm{CsCl}$ density gradient fractions were further purified using an RNeasy kit (QIAGEN) according to the manufacturer's instructions. Quantification of the DWV and GFP RNA and actin mRNA loads in individual honey bee pupae and associated Varroa mites were quantified by reverse transcription-quantitative PCR (RT-qPCR), which included cDNA synthesis using random hexanucleotides. RT-qPCR and cloning of the RT-PCR fragments were carried out essentially as previously described [14], using primers listed in Table S1. Statistical analyses of viral data, using log-transformed copy numbers of DWV and GFP RNA per pupa, were conducted by using the paired-sample T-test, and one-way analysis of variance (Figure 2; Table S3), and by one-way analysis of variance (ANOVA) followed by a Tukey post-hoc test to identify the differences between the groups (Figure S2).

\subsection{Detection of GFP and DWV Structural Proteins in Honey Bees by Western Blot and Immunofluorescence Microscopy}

Western blot analysis was carried out using a WesternBreeze kit (Invitrogen), following the manufacturer's instructions. The aliquots of homogenized honey bee pupae protein $(50 \mu \mathrm{g})$ or $20 \mu \mathrm{L}$ aliquots of $\mathrm{CsCl}$ gradient were heated at $95{ }^{\circ} \mathrm{C}$ for $10 \mathrm{~min}$ in a sodium dodecyl sulfate (SDS) sample buffer (WesternBreeze; Invitrogen) and separated on SDS 4\%-12\% Bis-Tri -polyacrylamide gel electrophoresis gels (Invitrogen). Polyclonal rabbit anti-GFP antibodies (ThermoFisher, Catalogue number PA1-980A) or rabbit polyclonal antisera raised to DWV virus particles purified using CsCl gradient centrifugation [15] were used to detect eGFP and DWV structural proteins of DWV, respectively. The leaf tissue of Nicotiana benthamiana, agroinfiltrated with the construct expressing $27 \mathrm{kDa}$ monomeric GFP [16], was used as a positive control for GFP. For visualization of eGFP expression in the injected pupae, the pupae at $72 \mathrm{hpi}$ were dissected and the hemolymph samples (approximately $30 \mu \mathrm{L}$ per pupae) with cells and fragments of tissue were collected, fixed with $500 \mu \mathrm{L}$ of $4 \%$ paraformaldehyde in PBS for $5 \mathrm{~min}$ at room temperature, centrifuged at $2500 \mathrm{rpm}$ in Eppendorf centrifuge for $2 \mathrm{~min}$ and 
washed three times with $500 \mu \mathrm{L}$ of PBS supplemented with $0.05 \%$ Tween-20. For DWV immunostaining, the cells were incubated with rabbit antisera raised against DWV particles [15] overnight at $+4{ }^{\circ} \mathrm{C}$; the Alexa Fluor 647-labelled goat anti-rabbit antibodies (Invitrogen, Catalogue number A11008) were used. Fluorescent microscopy was carried out using a Zeiss AX10 compound microscope (Zeiss Model Imager M2) fitted with and Axoio.M2 with camera 105. Visualization was carried out using the following excitation/emission wavelengths: GFP -488 nm/520 nm, 4' , 6-diamidino-2-phenylindole (DAPI) -359 nm/461 nm, Alexa Fluor 647-651 nm/676 nm.

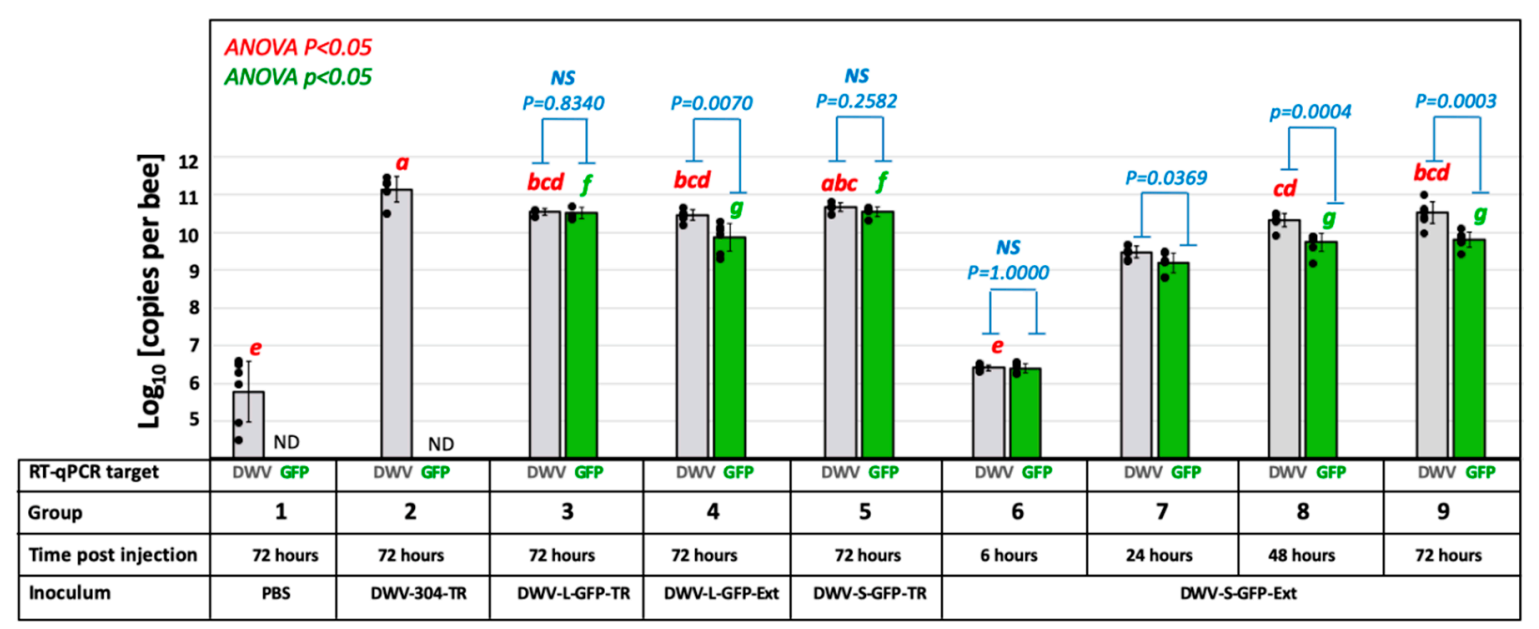

Figure 2. Replication of the GFP-tagged DWV in honey bee pupae. Average copy numbers of DWV and GFP RNA per pupa revealed by RT-qPCR are shown as the light grey and green graphs, respectively, the error bars show \pm standard deviation (SD), black dots show DWV RNA or GFP RNA copy numbers in individual pupae. Treatments are shown below the graphs; Inocula: PBS-phosphate buffered saline; suffix -TR, in vitro RNA transcript; suffix -Ext, filtered extract from the pupae infected with the corresponding in vitro RNA transcript. Red letters above the bars indicate significantly and non-significantly different groups (ANOVA, $P<0.05$ ). Blue bars-the statistical significance of the DWV and GFP copy numbers within the same group NS-non-significant (ANOVA $P>0.05$ ).

\section{Results}

\subsection{Design of GFP-Tagged DWV cDNA Constructs}

A full-length infectious cDNA clone of a US strain of wild-type DWV type A, pDWV-304 [11], was used to design two recombinant DWV constructs expressing enhanced GFP, pDWV-L-GFP and pDWV-S-GFP (Figure 1, Text S1, Text S2). In both constructs, the eGFP-coding sequence was inserted between the sequences coding for the leader protein (LP) and the structural virus protein 2 (VP2)-coding sequences in the frame to allow translation of ORF with eGFP inserts (Figure 1). The C-terminus of eGFP was linked to the amino acid position "minus 6" of the proposed proteolytic cleavage peptide between the LP and VP2 (VQAKPEMDNPNPG), which is tentatively targeted by DWV 3C protease [10] The N-terminus of eGFP was fused to the LP in two configurations: either via a proposed full proteolytic cleavage peptide VQAKPEMDNPNPG present at the LP-VP2 border in the clone pDWV-L-GFP, where proteolysis takes place between the glutamate and methionine residues $[10,17]$, or via a shorter peptide, VQAKPE, corresponding to the proposed C-terminus of the LP after proteolysis [10,17] (Figure 1). In the case of the pDWV-S-GFP construct, the linking peptide did not include the complete proposed proteolytic site, and therefore, this construct was expected to express LP-GFP as a fusion protein. In both egfp-tagged DWV constructs, proteolytic processing of the viral polypeptide between eGFP and VP2 was expected to take place, ensuring the generation of wild-type structural viral proteins required for the encapsidation of DWV RNA. 


\subsection{Infectivity the cDNA Clone-Derived GFP-Tagged DWV in Honey Bee Pupae}

Cell cultures are commonly used for studying the clone-derived RNA viruses of vertebrates and invertebrates, but this option is not readily applied to cloned DWV. Honey bee cell cultures suitable for work with DWV are not readily available. A recently reported honeybee cell line is infected already with DWV [18] and no other existing invertebrate cell cultures support DWV replication. Therefore, we injected honey bee pupae with viral in vitro RNA transcripts to recover and study recombinant clone-derived DWV, as reported previously [11]. In vitro RNA transcripts from the constructs pDWV-L-GFP and pDWV-S-GFP were infectious when injected into honey bee pupae intra-abdominally. Quantification of egfp target by RT-qPCR (Figure 2, Groups 3 and 5, GFP; Table S2) showed that at $72 \mathrm{~h}$ post injection (hpi) the pupae injected with pDWV-L-GFP and pDWV-S-GFP had high statistically identical $(P=0.802, \mathrm{df}=6$, ANOVA; Table S3) levels of genomic RNAs of DWV-L-GFP, ranging from 10.37 to $10.72 \log _{10}$ genome equivalents (GE) per pupa, and DWV-S-GFP, ranging from 10.33 to $10.66 \log _{10}$ GE per pupa (Figure 1, Groups 3 and 5; Table S2). Levels of the in vitro transcript-derived egfp-tagged viruses at $72 \mathrm{dpi}$ were far higher than those previously shown for of mutagenized non-replicating DWV in vitro transcripts [11] and control buffer injection (Figure 2, Group 1) ca. 5 to $7 \log _{10}$. Importantly, RT-qPCR revealed that the copy numbers of the egfp target (quantification of the genomes with the egfp insert only) and the DWV target (quantification of all DWV genomes, with and without egfp) were not significantly different in the pupae injected with the recombinant egfp-tagged DWV transcripts (Figure. 2; Group 3: $P=0.834$, df $=5$; Group 5: $P=0.258, \mathrm{df}$ = 7; ANOVA; Table S3). This showed that most of the recovered virus genomes in the pupae injected with the DWV-L-GFP and DWV-S-GFP in vitro transcripts retained egfp, and the presence of $717 \mathrm{nt}$ egfp insert (approximately 7\% of the DWV RNA) between the genes for LP and VP2 did not prevent replication of the modified viruses, i.e., by disrupting potential RNA elements essential for virus RNA replication [19].

Quantification of the DWV target showed that in the pupae injected with non-modified DWV in vitro RNA transcript from pDWV-304 the virus replicated to high levels (range: 10.51 to $11.47 \log _{10}$ GE/pupa; $11.14 \pm 0.338 \log _{10}$ GE/pupa, mean \pm SD, by 72 hpi; Table S2), which were significantly higher (four-fold) than in the DWV-L-GFP transcript-injected insects $(P=0.0398, \mathrm{df}=7$, ANOVA; Figure 2, Groups 2 and 3). Compared to the DWV-S-GFP transcript injection group, the levels of unmodified DWV-304 were three-fold higher, though this difference was not significant $(P=0.0505, \mathrm{df}=8, \mathrm{ANOVA}$; Figure 2, Groups 2 and 5). The levels of DWV in the control phosphate buffered saline (PBS)-injected pupae (Figure 2, Group 1, range: 4.48 to $6.59 \log _{10}$ GE/pupa; $5.79 \pm 0.801 \log _{10}$ GE/pupa; mean \pm SD) were significantly lower (tens of thousands of times) compared to those in any of the transcript-injected groups (Figure 2; Table S3, $P<0.0001$, ANOVA). As expected, the egfp RNA was not detected in the buffer control (PBS)- and the DWV-304-injected bees (Figure 2, Groups 1 and 2). Reduced levels of egfp-tagged DWV compared to the unmodified DWV-304, could reflect a fitness cost to the virus from increasing the genome size by $7 \%$ with a non-essential sequence.

To test whether expression of eGFP occurred in the pupae injected with the in vitro transcripts from the egfp-tagged DWV constructs, at $3 \mathrm{dpi}$, the pupal extracts were subjected to Western blot analysis with anti-GFP antibodies (Figure 3A). Control pupae injected with the buffer (PBS) or the wild-type DWV transcript (Figure 3A, lanes 4, 5), also at $72 \mathrm{hpi}$, were also included alongside the extracts from plant (Nicotiana benthamiana) tissues expressing monomeric GFP of $27 \mathrm{kDa}$ (Figure 3A, Lanes 2, 3) [16]. 
A

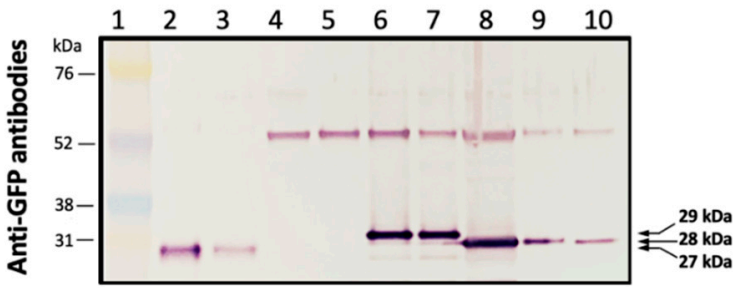

B

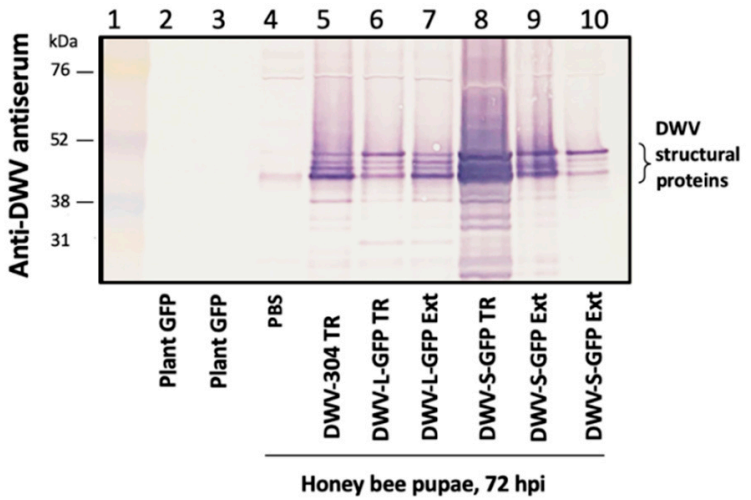

Figure 3. Expression of GFP in honey bee pupae from DWV vector. Western blot analysis using (A) anti-GFP antibodies, and (B) antiserum to DWV virus particles. Protein molecular weight markers (lane 1); lanes 2 and 3, GFP transiently expressed in Nicotiana benthamiana plants (lanes 2 and 3); honey bee pupae, $72 \mathrm{hpi}$, injected with PBS buffer (lane 4), DWV-304 in vitro RNA transcript (lane 5); DWV-L-GFP in vitro RNA transcript (lane 6); DWV-L-GFP filtered extract (lane 7); DWV-S-GFP in vitro RNA transcript (lane 8); DWV-S-GFP filtered extract (lanes 9 and 10). The expected molecular weights of GFP peptides are shown.

The Western blot analysis with anti-GFP antibodies clearly showed the accumulation of the $29 \mathrm{kDa}$ GFP protein in pupae injected with the DWV-L-GFP transcript, indicating accumulation of fully processed free eGFP. Surprisingly, in the case of DWV-S-GFP transcript accumulation of the $28 \mathrm{kDa}$ GFP rather than LP-GFP fusion was observed (Figure 3A, lane 8), indicating that proteolytic cleavage of the shorter version of the LP-GFP linking peptide also took place. The LP-GFP linking peptide, in the case of DWV-S-GFP, possibly led to the cleavage between glutamate and glycine residues of the peptide VQAKPEGAP, the glutamate-glycine cleavage site that was demonstrated for the DWV peptide linking VP1 and VP3 [10,17]. The observed differences of the molecular weight of DWV vectors compared to free monomeric GFP in the plant tissue controls (28 kDa for DWV-S-GFP, $29 \mathrm{kDa}$ for DWV-L-GFP, and $27 \mathrm{kDa}$ for plant-expressed free GFP) was probably the result of the residual peptides left after the proteolytic cleavage of the eGFP from the viral polyprotein and was in good agreement with their molecular weight predictions of $27.97 \mathrm{kDa}, 28.69 \mathrm{kDa}$ and $26.93 \mathrm{kDa}$, respectively (Figure S1). Importantly, the Western blot analysis with anti-GFP antibodies showed the absence of detectable quantities of polypeptides heavier than the expected monomeric eGFP, which could be the result of the accumulation of the eGFP fusions with LP and structural VP2 proteins (Figure 3A). The visible band with molecular weight about $55 \mathrm{kDa}$ was present not only in the case of DWV-L-GFP and DWV-S-GFP, but also in the case of the PBS-injected pupae with low DWV levels and the DWV-304 injected with high DWV levels (Figure 3A,B). This indicated complete proteolytic processing of the GFP-VP2 peptide in the case of both DWV-L-GFP and DWV-S-GFP (Figure 3A) suggesting that maturation of the structural proteins capable of packaging DWV genomic RNA took place during the replication of the egfp-tagged DWV.

\subsection{Analysis of Encapsidation of the egfp-Tagged DWV RNA Genomes}

DWV genomic RNAs with the egfp insert were approximately 7\% longer than wild-type DWV RNA (Text S2), potentially increasing the length of the modified genomic RNA beyond the RNA size 
limit for encapsidation. In addition, the insertion of a foreign sequence might potentially disrupt yet unknown RNA signals required for the formation of stable virus particles [19,20]. Therefore, it was necessary to determine if stable virus particles were formed in honey bee pupae infected with the egfp-tagged DWV and to compare them with wild-type DWV particles. To do this, we carried out cesium chloride $(\mathrm{CsCl})$ gradient centrifugation of the filtered extracts from honey bee pupae 72 hpi with the transcripts from the cDNA clones DWV-S-GFP and DWV-304 (unmodified DWV). Following ultracentrifugation, the fractions with densities ranging from $1.22 \mathrm{~g} / \mathrm{cm}^{3}$ to $1.58 \mathrm{~g} / \mathrm{cm}^{3}$ were collected and the copy numbers of DWV and GFP RNA targets were quantified for each fraction by RT-qPCR (Figure 4A,B).

A

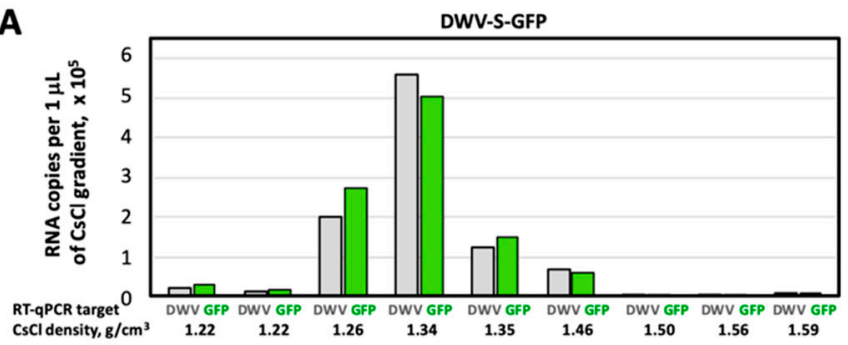

B
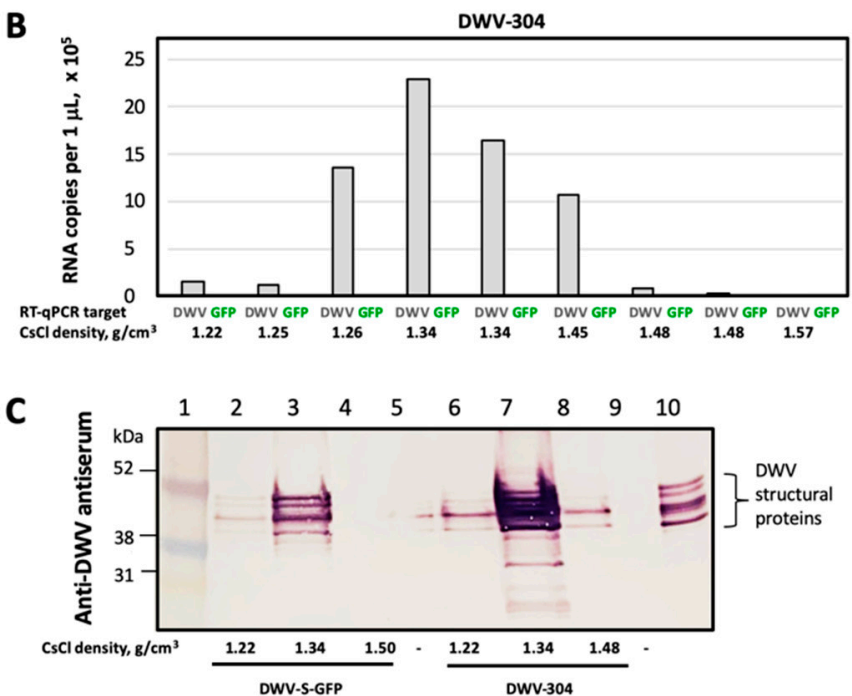

Figure 4. Encapsidation of the DWV-S-GFP genomic RNA. Virus particles accumulated in the honey bee pupae infected with (A) DWV-S-GFP and (B) DWV-304 in vitro RNA transcripts, 72 hpi were separated by $\mathrm{CsCl}$ buoyant density gradient centrifugations. The DWV and GFP RNA levels were quantified by RT-qPCR in the density gradient fractions indicated shown below. (C) Western blot analysis of the gradient fractions using antiserum to DWV virus particles. Lane 1, molecular weight standards; lanes 2-4, DWV-S-GFP CsCl gradient fractions; lanes 6-8, DWV-304 CsCl gradient fractions; lane 10, pupae infected with DWV-S-GFP; lanes 5 and 9, empty; lane 10 - extract from DWV-infected honeybee tissue.

We found that in the case of DWV-S-GFP, the $1.34 \mathrm{~g} / \mathrm{cm}^{3}$ buoyant density fraction showed the highest levels of both GFP and DWV targets (Figure 4A). In the case of DWV-304, the highest level of DWV RNA genome was also in the same $1.34 \mathrm{~g} / \mathrm{cm}^{3} \mathrm{CsCl}$ gradient fraction (Figure 4B), which was within the range of the predicted buoyant density for viruses of the family Iflaviridae in $\mathrm{CsCl}$ $\left(1.29-1.38 \mathrm{~g} / \mathrm{cm}^{3}\right)$ [21]. In addition, Western blot analysis with the antisera against DWV virus particles confirmed the highest load of the DWV capsid proteins in the fractions with the highest levels of DWV for both DWV-304 and DWV-S-GFP (Figure 4C). These results showed that the DWV RNA genome with a $750 \mathrm{nt}$ insertion (717 nt egfp gene and the sequences coding for duplicated flanking peptides) was encapsidated into particles with a stability and buoyant density similar to those of unmodified DWV. 
Encapsidation of DWV genomes with egfp inserts, both DWV-L-GFP and DWV-S-GFP, was further confirmed by infectivity of the filtered tissue extracts prepared from the transcript-infected honeybee pupae sourced at $72 \mathrm{hpi}$. Extracts selected for injection had nearly equal copy numbers of DWV and GFP RNA by RT-qPCR (Figure 2, Groups 3 and 5), indicating that they mostly contained the recombinant DWV genomes with the egfp inserts. In the case of DWV-S-GFP, an extract from the same transcript-infected pupae was used in $\mathrm{CsCl}$ buoyant density analysis (Figure 4A). Honey bee pupae injected with doses of $7 \log _{10}$ GE of DWV-L-GFP or DWV-S-GFP showed the development of recombinant virus infections. According to GFP quantification at $72 \mathrm{hpi}$, the levels of DWV-L-GFP ranged from 9.31 to $10.29 \log _{10} \mathrm{GE} /$ pupa $(9.87 \pm 0.364 \log 10 \mathrm{GE} /$ pupa; mean $\pm \mathrm{SD})$, and the levels of DWV-S-GFP ranged from 9.42 to $10.12 \log _{10}$ GE/pupa $(9.81 \pm 0.200 \log 10 \mathrm{GE} /$ pupa; mean \pm SD), Figure 2, Groups 5 and 10. There were no significant differences between the levels of DWV-L-GFP and DWV-S-GFP $(P=0.275, \mathrm{dF}=12$; Table S3).

We further confirmed the replication of recombinant DWV genotypes with intact egfp inserts in the pupae injected with DWV-L-GFP and DWV-S-GFP-derived extracts by Western blot analysis with the anti-GFP antibodies. Specifically, at 72 hpi monomeric eGFP bands of the same size were observed in both the extract-injected pupae and in the pupae injected with corresponding transcripts (Figure 3A, DWV-L-GFP: lanes 7, 8; DWV-S-GFP; lanes 8-10).

\subsection{Expression of eGFP Reporter from the DWV RNA Vectors}

Fluorescence microscopy was used to assess eGFP expression in honey bee pupae injected with the DWV-L-GFP and DWV-S-GFP filtered extracts at $72 \mathrm{dpi}$. Individual hemolymph cells and tissue fragments, possibly fragments of the immature intestinal epithelium, of the pupae injected with DWV-L-GFP (Figure 5A,B) and DWV-S-GFP (Figure 5C,D; Figure 6C,D) showed strong GFP fluorescence, which was not observed in the case of buffer (PBS) and the unmodified DWV (DWV-304) injections (Figure 6A,B).

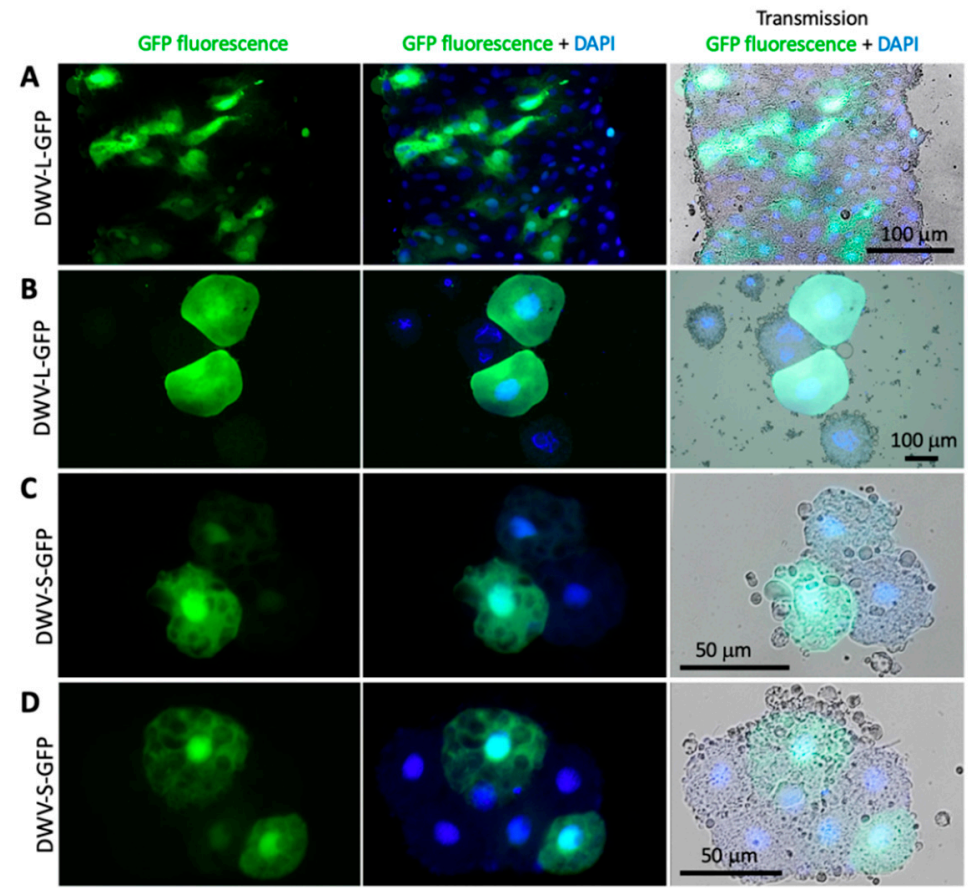

Figure 5. Localization of GFP in the honey bee cells infected with GFP-tagged DWV. Fluorescent microscopy of the cells of the pupae $72 \mathrm{~h}$ post injection with the filtered extracts containing $10^{7}$ genome equivalents (GE) of DWV-S-GFP (A,B) or DWV-S-GFP (C,D). Left panels, GFP fluorescence (green); middle panels, GFP fluorescence (green) and DAPI nuclear DNA staining (blue); right panels, microscopy transmission images combined with GFP fluorescence and DAPI staining. 


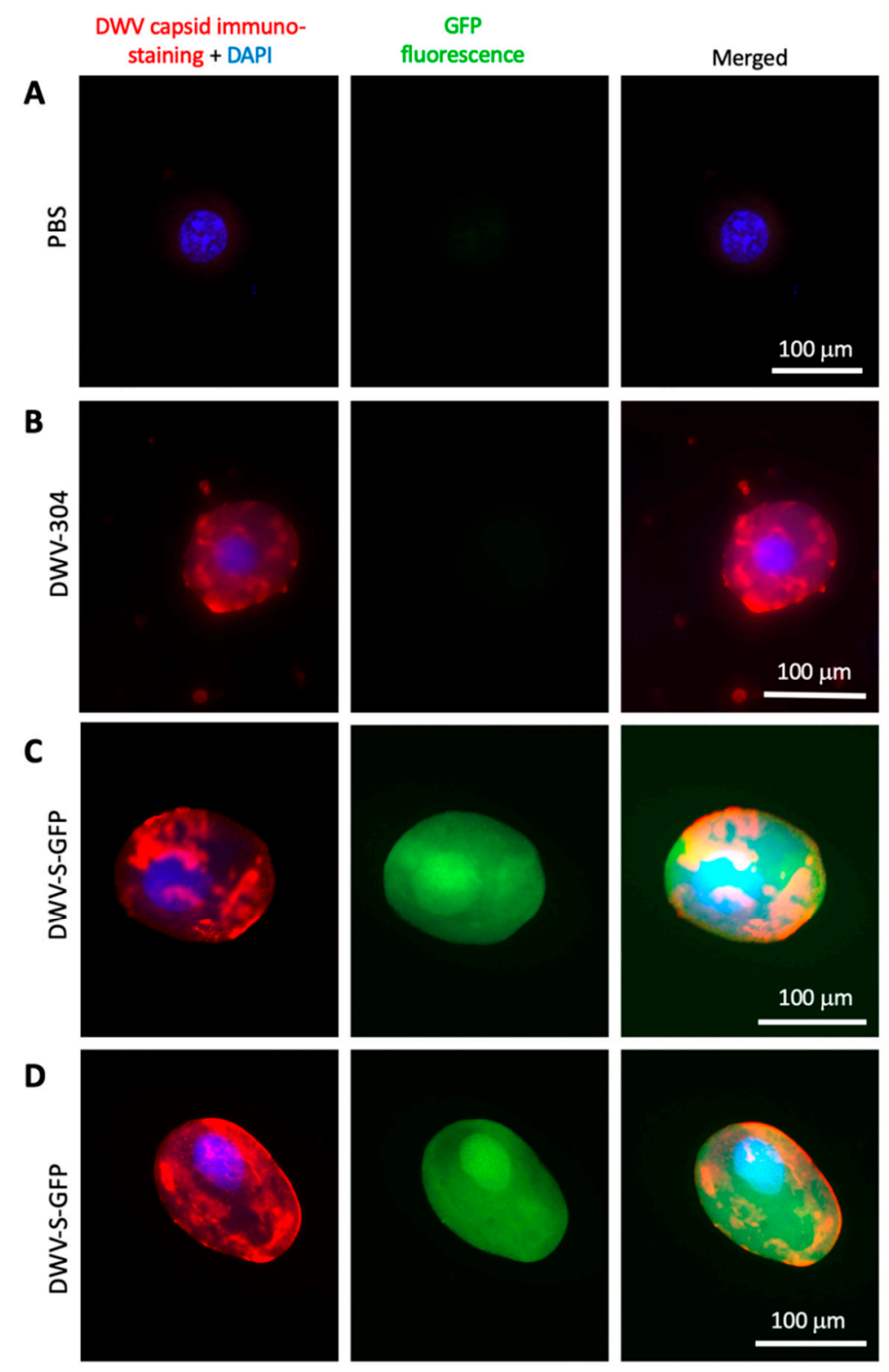

Figure 6. Localization of GFP and DWV structural proteins in the honey bee cells infected with GFP-tagged DWV. Fluorescent microscopy of the cells of the pupae $72 \mathrm{~h}$ post injection with PBS control (A), the filtered extracts containing $10^{7}$ genome equivalents of DWV-304 (B), or DWV-S-GFP (C,D). Left panels, immunostaining of the DWV capsids with anti-DWV antisera, Alexa Fluor 647 (red) and DAPI nuclear staining (blue); middle panels, GFP fluorescence (green); right panel, GFP fluorescence combined with Alexa Fluor 647 and DAPI staining.

The GFP fluorescence in the case of the egfp-tagged DWV variants was observed in both cytoplasm and nucleoplasm as confirmed by the co-localization of the GFP and DAPI fluorescence (Figure 6C,D), which was in agreement with previously reported localization of free GFP in insect cells [22]. Such localization of eGFP expressed from DWV vectors confirmed our Western blot analysis findings (Figure 3A) that complete proteolytic excision of eGFP from the viral polyprotein occurred. Fluorescent microscopy of the groups of cells in the case of DWV-L-GFP and DWV-S-GFP injections showed the presence of these cells with different levels of GFP expression in the same tissue fragment, which was evident from the DAPI fluorescence and transmission light microscopy (Figure 5). We also demonstrate, by using immunofluorescent microscopy with antibodies against the DWV capsid, that GFP was expressed in DWV-infected cells (Figure 6C,D). Immunofluorescence microscopy revealed that DWV 
capsid proteins were forming aggregates in the cytoplasm, while GFP was diffusely distributed in both cytoplasm and nucleoplasm in the same cells (Figure 6C,D).

\subsection{Genetic Stability of egfp-Tagged DWV RNA}

Quantification of the eGFP and DWV RNA targets in pupae injected with extracts containing $10^{7}$ GE of recovered DWV-L-GFP or DWV-S-GFP showed that at 72 dpi DWV copy numbers were significantly higher $(P<0.05$, ANOVA) than those of GFP indicating accumulation of DWV variants without egfp insert. Both egfp-tagged DWV variants showed similar levels of GFP and DWV at 72 hpi (Figure 2, Groups 4 and 9, GFP: $P=0.0 .715393, \mathrm{dF}=12$; DWV: $P=0.663399, \mathrm{dF}=11, \mathrm{ANOVA}$ ), and similar difference between DWV and GFP copy numbers in individual pupae (Figure S3, Groups 4 and 9, $P=0.1578, d F=12$, ANOVA). In the case of DWV-S-GFP injection, pupae were also sampled at 6, 24 and 48 hpi, showing non-significant differences between DWV and GFP copy numbers within each time group at 6 and $24 \mathrm{hpi}$, while at 48 dpi the copy number of DWV RNA significantly exceeded that of the GFP RNA (Figure 2, Groups 6, 7,8). Such differences suggest that in addition to the egfp-tagged DWV, injected pupae contained DWV without these inserts.

To determine the nature of the deletions in the DWV-S-GFP progeny, we amplified the section that included the egfp insert by RT-PCR using the primers flanking the egfp insert (Table S1) in the pooled DWV-S-GFP extract-injected pupae at $72 \mathrm{hpi}$ and cloned the heterologous fragments with egfp deletions into a plasmid vector. A sample of clones with inserts of different sizes was sequenced and we found different deletions of the 717 nt eGFP-coding sequence ranging from 357 to 438 nt (Figure 7A), Notably, in the case of all deletions, the ORF was restored, which was essential for translation of DWV RNA.

We tested whether the accumulation of DWV variants without the egfp insert was the result of the amplification of background DWV present in the recipient pupae at low levels (Figure 2, Group 1) or the accumulation of the DWV-L-GFP and DWV-S-GFP progeny with deletion of in the egfp gene. To do this, we determined if the PacI restriction site (Figure 1) was present in the $5^{\prime}$ IRES of the DWV progeny. The PacI sequence was absent in the wild-type DWV strains, but was introduced to the 5' IRES sequence of the parental DWV-304 clone [11] and was retained in both DWV-L-GFP and DWV-S-GFP; therefore, it could be used as a genetic marker to distinguish between wild-type DWV and cDNA clone-derived DWV. We demonstrated complete PacI digestion of the RT-PCR fragments amplified using samples of 72 hpi pupa injected with DWV-S-GFP and DWV-L-GFP inocula, but not with the wild-type DWV (Figure 7B), which indicated that all DWV genomes, including those without the $e g f p$ insert, were clone-derived.

When the DWV and GFP GE numbers were plotted on a two-dimensional graph (Figure 7C, $X$ axis - $\log _{10}$ DWV GE per pupae; $Y$ axis - $\log _{10}$ GFP GE per pupae), it became obvious that the proportion of the mutants with deletions of egfp was increasing with time. The DWV/GFP copy number ratio per injected honey bee was increasing with time after infection (Figure 7D). We estimate that the observed rate of accumulation in progeny with egfp deletions could be explained by an approximate $10 \%$ chance of such a deletion event per RNA replication event (Figure 7C, dotted projection lines). Notably, only genomes with deletion events within egfp inserts that resulted in the generation of a restored viral ORF were viable and were fixed in the viral population. Despite the accumulation of such deletion mutants, RT-qPCR results suggest that between 6 hpi and 72 hpi the load of the egfp-tagged virus had an average increased of approximately 2500, ranging from 1030 to 5196 times in individual pupa, compared to average GE counts at 6 hpi (Figure 2, Groups 6 and 9; Figure 7C, 6 hpi vs 72 hpi), clearly indicating strong infectivity of DWV genomes with the inserted foreign sequence (Figure 1). These results are in good agreement with the detection of GFP expression by fluorescent microscopy of the recombinant DWV extract-infected pupae (Figures 5 and 6). 
A DWV-S-GFP
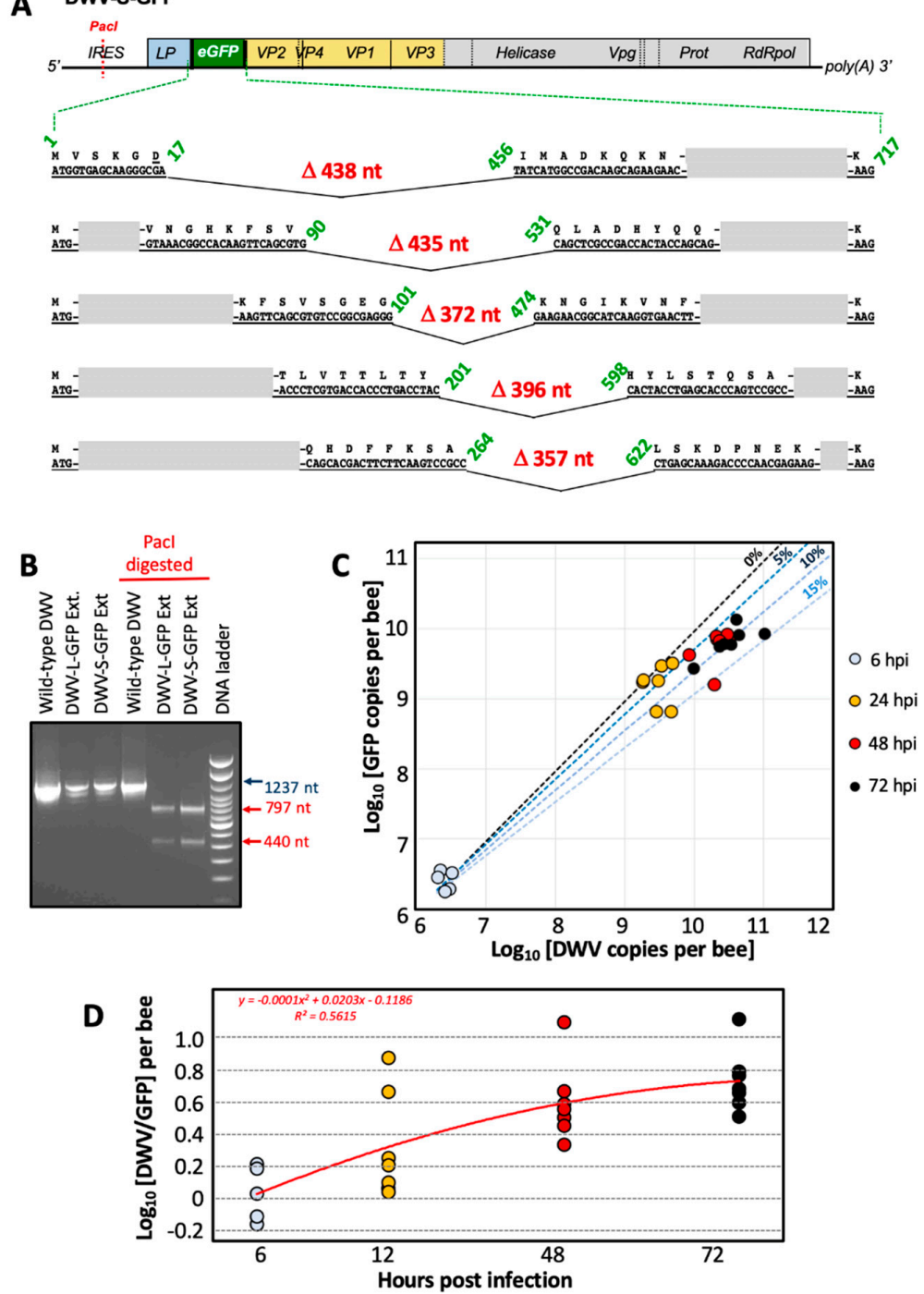

Figure 7. Genetic stability of the GFP-tagged DWV. (A) Deletions of the GFP-coding sequence in the DWV-S-GFP progeny accumulated in a honey bee pupae $72 \mathrm{~h}$ post injection (hpi) with the virus extract. The RT-PCR fragments were generated using the primers flanking GFP insert and cloned into a plasmid vector. Shown are the nucleotide sequences and the corresponding amino acid sequences adjacent to the deletion breakpoints, the numbers indicate the positions of the GFP sequence joined in the deletants and the sizes of deletions. The integrity of the viral ORF was restored in the case of all deletions. (B) Analysis of the 5' RT-PCR region. The RT-PCR fragments corresponding to the $5^{\prime}$ of DWV were produced using the combined samples of the pupae injected with DWV-L-GFP and DWV-S-GFP extracts at $72 \mathrm{hpi}$, pupae with the wild-type DWV was used as a control. The RT-PCR fragments were digested with PacI restriction enzyme, the site was present only in the cDNA clone-derived DWV. (C) Copy numbers of DWV (X-axis) and GFP ( $Y$-axis) RNA targets revealed by RT-qPCR in individual honey bee pupae 6, 24, 48 and 72 hpi with the virus extracts containing 107 genome equivalents of DWV-S-GFP. Dashed lines indicate projected ratios of DWV and GFP with $0 \%, 5 \%, 10 \%$ and $15 \%$ probability of generation deletion mutant during genome replication cycle starting from the average copy numbers observed at 6 hpi. (D) Temporal dynamics of the ratio between the copy number of DWV and GFP in the honey bee pupae infected with DWV-S-GFP. Log-transformed DWV/GFP ratio per individual pupae stratified according to time post inoculation. Regression analysis revealed a significant relationship $(R 2=0.5614)$ between the time after infection and the DWV/GFP ratio. Red line-quadratic regression curve over time, $R 2=0.5615$. 


\section{Discussion}

In this study, we designed and evaluated DWV-based virus vectors, the first constructed using the genome of the RNA virus naturally infecting honey bees [10], and demonstrated their ability to achieve high levels of expression of the eGFP reporter in honey bees. DWV is the major virus pathogen of honey bees and is associated with increased colony losses worldwide that have affected pollination services and food security [23,24]. DWV belongs to the family Iflaviridae (order Picornavirales), which includes 15 recognized and 18 pending virus species infecting a wide range of insects [21]. The real diversity and distribution of this virus group is likely much wider, and therefore, it is likely to have a great impact on many invertebrate species. For example, among the 1445 novel viruses discovered in a metagenomic analysis of viromes of 220 invertebrate species [25], approximately 400 of them belong to Picorna-Calici clade, some of which are likely to be the members of the order Picornavirales, including approximately 50 showing high homology with the previously identified iflaviruses. Despite a growing number of sequenced RNA viruses in invertebrates, there are significant gaps in the knowledge of their biology and interactions with their host(s). Therefore, the use of novel DWV vector constructs, in particular those expressing fluorescent reporters (e.g., eGFP) allowing non-invasive monitoring of the infection development in live cells and tissues, could provide novel insights into many viruses infecting invertebrates. Using DWV variants that express the fluorescent eGFP reporter only in the cells where viral replication takes place would facilitate precise identification of routes of viral infection, development and spread within honeybees of different stages infected either by injection or orally by pinpointing infected cells within the insect. Additionally, the use of such DWV constructs could be used to investigate the host range of the virus by monitoring DWV replication, manifested by GFP expression, at the single-cell level.

We demonstrated that the DWV RNA genome could be used to express a $29 \mathrm{kD}$ foreign protein (eGFP) when the egfp gene was inserted into the viral ORF in the frame between the sequences coding for the LP and VP2 structural protein. The eGFP polypeptide was flanked with the peptides derived from the DWV LP-VP2 interface allowing the release of free eGFP following the cleavage by DWV 3C protease (Figure 1). The expression eGFP was demonstrated by the observation of GFP fluorescence in the DWV-L-GFP- and DWV-S-GFP-infected cells of honey bees by fluorescent microscopy (Figures 5 and 6), and by the detection of polypeptides of expected sizes in Western blots experiments using antibodies against GFP (Figure 3A). The recombinant DWV genomes replicated to high levels, approximately $10^{10}$ to $10^{11} \mathrm{GE}$ per pupae by $72 \mathrm{hpi}$ (Figure 2), which indicated that the insertion of the foreign gene did not disrupt any cis-RNA elements required for replication [19].

There was a possibility that the increase of DWV genomic RNA by $7 \%$ as a result of egfp insertion could affect the encapsidation of the viral RNA. It was previously demonstrated that an RNA size limit for encapsidation exists for single stranded RNA viruses with icosahedral symmetry of virus particles structurally resembling those of Iflaviruses, such as Turnip crinkle virus [26,27]. Additionally, potential RNA elements required for DWV RNA encapsidation could be disrupted $[19,20]$. Therefore, we used the $\mathrm{CsCl}$ density gradient centrifugation to test if the formation of stable DWV particles containing DWV genomic RNA with egfp inserts took place in in the pupae injected with the DWV-S-GFP and DWV-L-GFP in vitro RNA transcripts. We found that DWV genome with egfp insertion was packaged into stable virus particles and had the same physical properties as those containing unmodified DWV RNA (Figure 4).

One of the aims of this study was to develop a tagged DWV vector, which could be used for tracking virus replication in the cells and tissues of the DWV host(s). Indeed, we observed bright GFP fluorescence in the cells of the pupae infected with the extracts containing recovered DWV-L-GFP or DWV-S-GFP. Different cell-types showed GFP fluorescence, which was consistent with the previously reported presence of DWV throughout the honey bee body in various cell types [28]. Importantly, GFP fluorescence was observed in the pupae infected with the filtered tissue extracts, showing the suitability of using the DWV vector for large scale studies. In our experiment, we found that the amount of the recovered egfp-expressing recombinant virus inoculum generated in a single 
in vitro RNA transcript-injected pupa was sufficient for hundreds of pupal injections. Therefore, the eGFP-tagged DWV (USA strain of DWV type A) was designed in this study, including other cloned DWV-like viruses [29-31], which could also be tagged with a reporter gene in the way developed in this study. Such tagged DWV-like viruses could be an excellent tool to advance the understanding of the mechanisms of vectoring of DWV-like viruses by the Varroa mites [32] and the host range of DWV-like viruses in different bee species. Currently, there are conflicting reports on whether DWV-like viruses replicate in their Varroa mite vector [33-36] and the use of cloned DWV-like viruses expressing egfp during their replication would allow to determine if replication of any variants of DWV-like viruses occur in their Varroa mite vector and pinpoint the Varroa mite cells where replication could take place.

We also analyzed the genetic stability of our egfp tagged DWV constructs. While deletion of foreign genes from RNA virus vector genomes was reported previously [37,38], this was the first study of the genetic stability of a picorna-like RNA virus (Iflavirus) vector conducted in insects at the organism level. Our finding supports previous reports that the size of foreign insert dramatically affects the genetic stability of the recombinant virus therefore shorter inserts were more tolerated. For example, for a poliovirus-based vector (approximately $7500 \mathrm{nt}$ RNA genome), the constructs with inserts with a size below $282 \mathrm{nt}$ were more stable than those with $402 \mathrm{nt}$ or $744 \mathrm{nt}$ [37]. Interestingly, we found that some of the egfp deletion mutant genomes generated at 72 hpi in the course of DWV-S-GFP infection in the extract-injected bees still had 200-300 nt-long parts of the eGFP-coding sequence (Figure 7A). It is likely that the deletion of non-essential gene insertions, even if these were not interfering with virus replication or encapsidation, could be driven by higher competitiveness of a shorter RNA genome. Moreover, infection of an RNA virus in the host transgenically expressing one of the virus proteins rapidly results in the generation of the mutants of this virus with the deletion of the gene in the virus genome corresponding to the transgene, as was demonstrated for infection of the Tobacco etch virus (TEV) gene in plants expressing the TEV NIb replicase gene [39]. Longer size of genomic RNAs of DWV-S-GFP, DWV-L-GFP and even their deletion mutants (some of which still contained parts of GFP) might explain their reduced levels compared to parental non-modified DWV-304 at 72 hpi (Figure 2, Groups 2, vs. Groups 3, 4, 5, 9; DWV probes).

It should be emphasized that although the accumulation of the progeny with the deletion of non-essential egfp took place, this process was gradual and did not happen immediately. We showed that the egfp-tagged viruses replicated in the injected pupae, increased their loads thousands of times (Figure 2, Figure 7C), suggesting that even a non-essential gene in an RNA virus genome could be relatively stable (Figure 7C). It is a widely accepted view that gene exchanges between different virus groups and incorporation of host mRNA or their fragments occur during the RNA virus evolution $[40,41]$. These foreign inserts must be present in genomic RNA undergoing several rounds of replication events to allow further adjustment to the viral RNA genome context via mutagenesis. Our results suggest that even the foreign gene, which did not bring benefits to the virus, could be a part of an RNA genome for a prolonged time, allowing the possibility of mutagenesis. Importantly, the results indicate that such insertion(s) in the genome will not dramatically affect replication or other essential virus functions.

\section{Conclusions}

In this study, the DWV vectors were constructed using a full-length infectious cDNA clone of DWV [11] by inserting sequences coding for the egfp reporter gene using a novel arrangement, which has not been applied to constructing picorna-like vectors previously [2], and included the insertion of a foreign gene into DWV ORF between the sequences coding for the leader protein (LP) and the structural virus protein (VP2). This novel arrangement of the foreign gene insertion did not disrupt replication or encapsidation. While we have shown the value of this approach for the expression of the reporter protein, the same approach might be applied for expressing other proteins or RNA molecules important for functional studies or even for maintaining honey bee health. Indeed, the potential of 
genetically modified bacteria for providing disease-modulating dsRNA for honey bees has been shown recently [42], and it is quite possible that DWV-based vectors might be enlisted for the same role.

Supplementary Materials: The following are available online at http://www.mdpi.com/1999-4915/12/4/374/s1, Figure S1: The eGFP-coding sequences inserts in DWV vector, Figure S2: Replication of the GFP-tagged DWV in honey bee pupae analyzed using a one-way ANOVA followed by a Tukey post-hoc test, Figure S3: Accumulation of the egfp deletant genomes in individual honey bee pupae, Table S1: Primers and the synthetic gene used in this study, Table S2: Quantification of DWV and GFP RNA targets in individual pupae, Table S3: Statistical significance of the differences between DWV and GFP RNA copy numbers, Text S1. Design of pDWV-L-GPF and DWV-S-GFP, Text S2: Nucleotide sequences of the Deformed wing virus vector cDNA clones pDWV-L-GFP and pDWV-S-GFP.

Author Contributions: Conceptualization, E.V.R.; methodology, E.V.R., M.C.H., F.P.-F., R.L.H. and J.D.E.; validation, E.V.R and J.D.E.; formal analysis, E.V.R.; investigation, E.V.R., K.C., M.C.H. and F.P.-F.; resources, E.V.R., F.P.-F., R.L.H. and J.D.E.; data curation, E.V.R.; writing-original draft preparation, E.V.R. and J.D.E.; writing-review and editing, E.V.R., K.C., M.C.H., F.P.-F., R.L.H., Y.C. and J.D.E.; visualization, E.V.R., K.C. and R.L.H.; supervision, E.V.R, Y.C. and J.D.E.; project administration, J.D.E., Y.C. and E.V.R.; funding acquisition, E.V.R., Y.C. and J.D.E. All authors have read and agreed to the published version of the manuscript.

Funding: Research was funded by the United States Department of Agriculture (USDA) National Institute of Food and Agriculture (NIFA) grant number 2017-06481 (for E.V.R., Y.C. and J.DE.), ORISE fellowship (for F.P.-F.) and the USDA Animal and Plant Health Inspection Service (APHIS) agreement 19-8130-0745-IA (for J.D.E.) and APHIS Federal Award AP18PPQS\&T00C060 (for K.C.).

Acknowledgments: We thank Mike Simone-Finstrom (USDA Baton Rouge, Louisiana) for providing honey bees, Svetlana Folimonova (University of Florida) for GFP control and Zachary Lamas for the symptomatic honey bees infected with wild-type DWV.

Conflicts of Interest: The authors declare no conflict of interest. The funders had no role in the design of the study; in the collection, analyses or interpretation of data; in the writing of the manuscript, or in the decision to publish the results.

\section{References}

1. Mogler, M.A.; Kamrud, K.I. RNA-based viral vectors. Expert Rev. Vaccines 2014, 14, 283-312. [CrossRef] [PubMed]

2. Ylä-Pelto, J.; Tripath, L.; Susi, P. Therapeutic use of native and recombinant enteroviruses. Viruses 2016, 8, 57. [CrossRef] [PubMed]

3. Donson, J.; Kearney, C.M.; Hilf, M.E.; Dawson, W.O. Systemic expression of a bacterial gene by a Tobacco mosaic virus-based vector. Proc. Natl. Acad. Sci. USA 1991, 88, 7204-7208. [CrossRef] [PubMed]

4. Pogue, G.P.; Lindbo, J.A.; Garger, S.J.; Fitzmaurice, W.P. Making an ally from an enemy: Plant virology and the new agriculture. Annu. Rev. Phytopathol. 2002, 40, 45-74. [CrossRef] [PubMed]

5. Robertson, D. VIGS vectors for gene silencing: Many targets, many tools. Annu. Rev. Plant Biol. 2004, 55, 495-519. [CrossRef] [PubMed]

6. Oparka, K.J.; Boevink, P.; Santa Cruz, S. Studying the movement of plant viruses using green fluorescent protein. Trends Plant Sci. 1996, 12, 412-418. [CrossRef]

7. Nouri, S.; Matsumura, E.E.; Kuo, Y.W.; Falk, B.W. Insect-specific viruses: From discovery to potential translational applications. Curr. Opin. Virol. 2018, 33, 33-41. [CrossRef] [PubMed]

8. Dasgupta, R.; Cheng, L.-L.; Bartholomay, L.C.; Christensen, B.M. Flock house virus replicates and expresses green fluorescent protein in mosquitoes. J. Gen. Virol. 2003, 84, 1789-1797. [CrossRef]

9. Flenniken, M.L.; Andino, R. Non-specific dsRNA-mediated antiviral response in the honey bee. PLoS ONE 2013, 8, e77263. [CrossRef]

10. Lanzi, G.; de Miranda, J.R.; Boniotti, M.B.; Cameron, C.E.; Lavazza, A.; Capucci, L.; Camazine, S.M.; Rossi, C. Molecular and biological characterization of deformed wing virus of honeybees (Apis mellifera L.). J. Virol. 2006, 80, 4998-5009. [CrossRef]

11. Ryabov, E.V.; Childers, A.K.; Lopez, D.; Grubbs, K.; Posada-Florez, F.; Weaver, D.; Girten, W.; vanEngelsdorp, D.; Chen, Y.; Evans, J.D. Dynamic evolution in the key honey bee pathogen deformed wing virus: Novel insights into virulence and competition using reverse genetics. PLoS Biol. 2019, 17, e3000502. [CrossRef] [PubMed]

12. Cormack, B.P.; Valdivia, R.; Falkow, S. FACS-optimized mutants of the green fluorescent protein (GFP). Gene 1996, 173, 33-38. [CrossRef] 
13. Shi, X.; Harrison, R.L.; Hollister, J.R.; Mohammed, A.; Fraser, M.J.; Jarvis, D.L. Construction and characterization of new piggyBac vectors for constitutive or inducible expression of heterologous gene pairs and the identification of a previously unrecognized activator sequence in piggyBac. BMC Biotechnol. 2007, 7, 5. [CrossRef] [PubMed]

14. Ryabov, E.V.; Childers, A.K.; Chen, Y.; Madella, S.; Nessa, A.; vanEngelsdorp, D.; Evans, J.D. Recent spread of Varroa destructor virus-1, a honey bee pathogen, in the United States. Sci. Rep. 2017, 7, 17447. [CrossRef] [PubMed]

15. Fannon, J.M.; Ryabov, E.V. Iflavirus (Deformed Wing Virus). In Molecular Detection of Animal Viral Pathogens; Liu, D., Ed.; CRC Press: Boca Raton, FL, USA, 2016; pp. 37-46.

16. Kang, S.-H.; Bak, A.; Kim, O.-K.; Folimonova, S.Y. Membrane association of a nonconserved viral protein confers virus ability to extend its host range. Virology 2015, 482, 208-217. [CrossRef] [PubMed]

17. Škubník, K.; Nováček, J.; Füzik, T.; Přidal, A.; Paxton, R.J.; Plevka, P. Structure of deformed wing virus, a major honey bee pathogen. Proc. Natl. Acad. Sci. USA 2017, 114, 3210-3215. [CrossRef]

18. Carrillo-Tripp, J.; Dolezal, A.G.; Goblirsch, M.J.; Miller, W.A.; Toth, A.L.; Bryony, C.; Bonning, B.C. In vivo and in vitro infection dynamics of honey bee viruses. Sci. Rep. 2016, 6, 22265. [CrossRef]

19. Liu, Y.; Wimmer, E.; Paul, A.V. Cis-acting RNA elements in human and animal plus-strand RNA viruses. Biochim. Biophys. Acta 2009, 1789, 495-517. [CrossRef]

20. Sasaki, J.; Taniguchi, K. The 5-end sequence of the genome of Aichi virus, a picornavirus, contains an element critical for viral RNA encapsidation. J. Virol. 2003, 77, 3542-3548. [CrossRef]

21. Valles, S.M.; Chen, Y.; Firth, A.E.; Guérin, D.M.; Hashimoto, Y.; Herrero, S.; de Miranda, J.R.; Ryabov, E. ICTV Virus Taxonomy Profile: Iflaviridae. J. Gen. Virol. 2017, 98, 527-528. [CrossRef]

22. Liu, H.; Wei, C.; Zhong, Y.; Li, Y. Rice black-streaked dwarf virus minor core protein P8 is a nuclear dimeric protein and represses transcription in tobacco protoplasts. FEBS Lett. 2007, 581, 2534-2540. [CrossRef] [PubMed]

23. Martin, S.J.; Brettell, L.E. Deformed Wing Virus in Honeybees and Other Insects. Annu. Rev. Virol. 2019, 6, 49-69. [CrossRef] [PubMed]

24. Grozinger, C.M.; Flenniken, M.L. Bee Viruses: Ecology, Pathogenicity, and Impacts. Annu. Rev. Entomol. 2019, 64, 205-226. [CrossRef] [PubMed]

25. Shi, M.; Lin, X.D.; Tian, J.H.; Chen, L.J.; Chen, X.; Li, C.X.; Qin, X.C.; Li, J.; Cao, J.P.; Eden, J.S.; et al. Redefining the invertebrate RNA virosphere. Nature 2016, 540, 539-543. [CrossRef]

26. Qu, F.; Morris, T.J. Efficient Infection of Nicotiana benthamiana by Tomato bushy stunt virus is Facilitated by the Coat Protein and Maintained by p19 Through Suppression of Gene Silencing. MPMI 2002, 15, 193-202. [CrossRef]

27. Qu, F.; Morris, T.J. Encapsidation of Turnip Crinkle Virus Is Defined by a Specific Packaging Signal and RNA Size. J. Virol. 1997, 71, 1428-1435. [CrossRef]

28. Fievet, J.; Tentcheva, D.; Gauthier, L.; de Miranda, J.; Cousserans, F.; Colin, M.E.; Bergoin, M. Localization of deformed wing virus infection in queen and drone Apis mellifera L. Virol. J. 2006, 3, 16. [CrossRef]

29. Lamp, B.; Url, A.; Seitz, K.; Eichhorn, J.; Riedel, C.; Sinn, L.J.; Indik, S.; Köglberger, H.; Rümenapf, T. Construction and Rescue of a Molecular Clone of Deformed Wing Virus (DWV). PLoS ONE 2016, 11, e0164639. [CrossRef]

30. Posada-Florez, F.; Ryabov, E.V.; Heerman, M.C.; Chen, Y.; Evans, J.D.; Cook, S.C.; Sonenshine, S.C. A novel system for maintaining Varroa destructor mites on artificial diets and its application for studying mites as a vector for honey bee viruses. BioRxiv 2019. [CrossRef]

31. Gusachenko, O.N.; Woodford, L.; Balbirnie-Cumming, K.; Ryabov, E.V.; Evans, D.J. Deformed Wing Virus spillover from honey bees to bumble bees: A reverse genetic study. BioRxiv 2019. [CrossRef]

32. Rosenkranz, P.; Aumeier, P.; Ziegelmann, B. Biology and control of Varroa destructor. J. Invertebr. Pathol. 2010, 103, S96-S119. [CrossRef] [PubMed]

33. Yue, C.; Genersch, E. RT-PCR analysis of Deformed wing virus in honeybees (Apis mellifera) and mites (Varroa destructor). J. Gen. Virol. 2005, 86, 3419-3424. [CrossRef] [PubMed]

34. Santillán-Galicia, M.T.; Carzaniga, R.; Ball, B.B.; Alderson, P.G. Immunolocalization of deformed wing virus particles within the mite Varroa destructor. J. Gen. Virol. 2008, 89, 1685-1689. [CrossRef] [PubMed]

35. Gisder, S.; Aumeier, P.; Genersch, E. Deformed wing virus: Replication and viral load in mites (Varroa destructor). J. Gen. Virol. 2009, 90, 463-467. [CrossRef] 
36. Posada-Florez, F.; Childers, A.K.; Heerman, M.C.; Egekwu, N.I.; Cook, S.C.; Chen, Y.; Evans, J.D.; Ryabov, E.V. Deformed wing virus type A, a major honey bee pathogen, is vectored by the mite Varroa destructor in a non-propagative manner. Sci. Rep. 2019, 9, 12445. [CrossRef]

37. Dobrikova, E.Y.; Florez, P.; Gromeier, M. Structural determinants of insert retention of poliovirus expression vectors with recombinant IRES elements. Virology 2003, 311, 241-253. [CrossRef]

38. Chung, B.-N.; Tomas Canto, T.; Palukaitis, P. Stability of recombinant plant viruses containing genes of unrelated plant viruses. J. Gen. Virol. 2007, 88, 1347-1355. [CrossRef]

39. Tromas, N.; Zwart, M.P.; Forment, J.; Elena, S.F. Shrinkage of Genome Size in a Plant RNA Virus upon Transfer of an Essential Viral Gene into the Host Genome. Genome Biol. Evol. 2014, 6, 538-550. [CrossRef]

40. Koonin, E.V.; Dolja, V.V.; Krupovic, M. Origins and evolution of viruses of eukaryotes: The ultimate modularity. Virology 2015, 479, 2-25. [CrossRef]

41. Elena, S.F. Evolutionary transitions during RNA virus experimental evolution. Philos. Trans. R. Soc. B 2016, 371, 20150441. [CrossRef]

42. Leonard, S.P.; Powell, J.E.; Perutka, J.; Geng, P.; Heckmann, L.C.; Horak, R.D.; Davies, B.W.; Ellington, A.D.; Barrick, J.E.; Moran, N.A. Engineered symbionts activate honey bee immunity and limit pathogens. Science 2020, 367, 573-576. [CrossRef] [PubMed]

(C) 2020 by the authors. Licensee MDPI, Basel, Switzerland. This article is an open access article distributed under the terms and conditions of the Creative Commons Attribution (CC BY) license (http://creativecommons.org/licenses/by/4.0/). 